\title{
GELFAND-KIRILLOV DIMENSION OF MULTI-FILTERED ALGEBRAS
}

\author{
by JOSÉ GÓMEZ TORRECILLAS*
}

(Received 18th March 1997)

\begin{abstract}
We consider associative algebras filtered by the additive monoid $\mathbb{N}^{p}$. We prove that, under quite general conditions, the study of Gelfand-Kirillov dimension of modules over a multi-filtered algebra $R$ can be reduced to the associated $\mathbb{N}^{p}$-graded algebra $G(R)$. As a consequence, we show the exactness of the GelfandKirillov dimension when the multi-filtration is finite-dimensional and $G(R)$ is a finitely generated noetherian algebra. Our methods apply to examples like iterated Ore extensions with arbitrary derivations and "homothetic" automorphisms (e.g. quantum matrices, quantum Weyl algebras) and the quantum enveloping algebra of $\operatorname{sl}(v+1)$
\end{abstract}

1991 Mathematics subject classification: 16P90, 16W60, $16 \mathrm{~W} 50$.

\section{Introduction}

A useful technique to study the Gelfand-Kirillov dimension of an associative algebra $R$ over a field $k$ is to find a "finite-dimensional" filtration $\left\{F_{n}(R): n \in \mathbb{N}\right\}$ on $R$ with a simpler associated graded ring $G(R)$ (cf. [13, Ch. 8]). The most typical situation arises when $G(R)$ is commutative (the algebra $R$ is then called "somewhat commutative"). Some interesting examples of algebras, like the quantum coordinate algebras $\mathcal{O}_{q}\left(M_{n}(k)\right)$ of the $n \times n$-matrices over $k$ or the quantum universal algebras $U_{q}(s l(v+1))$, resist this tool. McConnell [12] found a finite-dimensional filtration on $\mathcal{O}_{q}\left(M_{n}(k)\right)$ with semicommutative associated graded algebra (i.e., this graded algebra has finitely many generators that commute up to nonzero scalars in $k$ ). In the case of the quantum enveloping algebra, he found an infinite dimensional $\mathbb{N}^{2}$-filtration with semicommutative associated ring for which some results from [14] could be applied. The key idea in the present work is that if we enlarge the size of the filtering monoid, then the infinite dimensions in the filtration can be avoided.

In Section 1 we state the notion of multi-filtration for rings and modules. The indexing of the filtrations uses a multi-index $\alpha \in \mathbb{N}^{p}$, where $\mathbb{N}^{p}$ is endowed with a total order compatible with the additive monoid structure. The associated $\mathbb{N}^{p}$-graded rings and modules are defined and, as an easy first application of these concepts, we prove a kind of Hilbert Basis Theorem (Theorem 1.5).

\footnotetext{
* Research supported by the grant PB94-0791 from the DGICYT.
} 
Section 2 is the core of the paper. Essentially, it is devoted to proving that if a $k$-algebra $R$ has a multi-filtration by finite-dimensional vector subspaces such that the associated graded ring $G(R)$ is a finitely generated $k$-algebra, then the Gelfand-Kirillov dimension can be computed on $G(R)$ (Theorem 2.8). Moreover, if $G(R)$ is finitely generated and noetherian (e.g. if $G(R)$ is semi-commutative), then the Gelfand-Kirillov dimension is exact on short exact sequences of finitely generated left $R$-modules (Theorem 2.10 and Corollary 2.12).

Section 3 is devoted to providing examples of finite dimensional multi-filtrations on some algebras. We include some types of iterated Ore extensions (in particular, iterated differential operator algebras [17], the algebras $H(p, \lambda)$ introduced in [1] and the multiparameter quantum Weyl algebras [11] are covered). The quantum universal enveloping algebra $U_{q}(\mathfrak{s l}(v+1))$ is also satisfactorily multi-filtered.

\section{Multi-filtrations}

Throughout this paper, $R$ will denote an associative unitary ring. Let $p$ be a positive integer. We consider $\mathbb{N}^{p}$ as additive monoid with the sum defined componentwise.

Definition 1.1. An admissible order " $\leq$ " on $\left(\mathbb{N}^{p},+\right)$ is a total order such that,

(a) $0=(0, \ldots, 0) \leq \alpha$ for every $\alpha \in \mathbb{N}^{p}$.

(b) For all $\alpha, \beta, \gamma, \in \mathbb{N}^{p}$ with $\alpha \leq \beta$ it follows $\alpha+\gamma \leq \beta+\gamma$.

Remark 1.2. By Dickson's Lemma (see, e.g., [2, Corollary 4.48]), admissible orders on $\mathbb{N}^{p}$ are good orders (i.e., any non empty subset of $\mathbb{N}^{p}$ has a first element).

From now on, fix an admissible order " $\leq$ " on $\left(\mathbb{N}^{p},+\right)$.

Definition 1.3. A multi-filtration on $R$ is a family $\left\{F_{y}(R) \mid \gamma \in \mathbb{N}^{p}\right\}$ of subgroups of the additive group of $R$ satisfying the following axioms.

1. $F_{y}(R) \subseteq F_{\delta}(R)$ if $\gamma \leq \delta$.

2. $F_{y}(R) F_{\delta}(R) \subseteq F_{y+\delta}(R)$.

3. $\bigcup_{a \in \mathbb{N}^{p}} F_{\alpha}(R)=R$.

4. $1 \in F_{0}(R)$.

The notion of multi-filtered ring appears in [6,7.4.2], although assuming a partial ordering in the additive monoid. The canonical bases found by Lusztig in [10] give a multi-filtration on the quantum universal enveloping algebra $U_{q}(g)$ of a semi-simple Lie algebra $\mathfrak{g}$ (see $[4,3.10]$ ). The filtering vector subspaces are not of finite dimension in this case, so that the arguments developed in the next section to study the GelfandKirillov dimension do not work in this case. However, we will show how to apply our techniques to $U_{q}(5 l(v+1))$ (see Example 3.4). 
In what follows, we assume that the ring $R$ is endowed with a fixed multifiltration.

Definition 1.4. A multi-filtration on a left $R$-module $M$ is a family $\left\{F_{y}(M) \mid \gamma \in \mathbb{N}^{P}\right\}$ of subgroups of $M$ satisfying the following axioms.

1. $F_{\gamma}(M) \subseteq F_{\delta}(M)$ if $\gamma \leq \delta$.

2. $F_{y}(R) F_{\delta}(M) \subseteq F_{y+\delta}(M)$.

3. $\bigcup_{z \in N^{p}} F_{a}(M)=M$.

The multi-filtered ring $R$ has an associated $\mathbb{N}^{p}$-graded ring defined as follows (see [16] and [15] for a computational approach to this notion). Given a multi-filtered left $R$-module $M$, and $\gamma \in \mathbb{N}^{p}$, write

$$
V_{\gamma}(M)=\bigcup_{\gamma^{\prime}<\gamma} F_{\gamma^{\prime}}(M)
$$

It is understood that $V_{0}=\{0\}$. Consider the additive group

$$
G_{y}(M)=F_{y}(M) / V_{y}(M),
$$

and define

$$
G(M)=\bigoplus_{y \in \mathbb{N}^{p}} G_{y}(M)
$$

For $r+V_{y}(R) \in G_{y}(R)$ and $m+V_{\delta}(M) \in G_{\delta}(M)$, define

$$
\left(r+V_{\gamma}(R)\right)\left(m+V_{\delta}(M)\right)=r m+V_{\gamma+\delta}(M) .
$$

If $M=R$, then we have a product in $G(R)$ which makes $G(R)$ into a $\mathbb{N}^{p}$-graded ring. Moreover, $G(M)$ becomes an $\mathbb{N}^{p}$-graded left $G(R)$-module.

In the case of $\mathbb{N}$-filtered rings, several properties can be lifted from $G(R)$ to $R$ (see e.g. [13]). From a heuristic viewpoint, this would be true for multi-filtered rings. The following is a relevant first example.

Theorem 1.5 (Hilbert Basis Theorem). Let $R$ be a multi-filtered ring. If $G(R)$ is left noetherian then $R$ is left noetherian.

Proof. For a left ideal $I$ of $R$,

$$
G(I)=\bigoplus_{y \in \mathbb{N}^{\prime}} \frac{\left(I \cap F_{y}(R)\right)+V_{y}(R)}{V_{y}(R)}
$$


is a graded left ideal of $G(R)$. It is enough to prove that if $I \subseteq J$ are left ideals of $R$ such that $G(I)=G(J)$, then $I=J$. Let $0 \neq r \in J$ and set $\alpha=\min \left\{\gamma \in \mathbb{N}^{p} \mid r \in F_{y}(R)\right\}$. Then $r \notin V_{x}(R)$ and, thus, $r+V_{x}(R) \in G(J)_{x}=G(I)_{x}$. There is $r_{x} \in I$ such that $r-r_{x} \in V_{\alpha}(R)$. It is clear that $r-r_{\alpha} \in J \cap V_{\alpha}(R)$, whence $r-r_{\alpha} \in J \cap F_{\beta}(R)$ for some $\beta<\alpha$. If $r-r_{\alpha} \neq 0$, then repeat the argument for $r-r_{\alpha}$ and, after a finite number of steps, we can write $r$ as a finite sum of elements of $I$. Certainly, $I=J$.

\section{Gelfand-Kirillov dimension}

Fix a commutative field $k$ and assume that $R$ is a $k$-algebra. First, while we introduce some notation, we will recall the notion of Gelfand-Kirillov dimension of finitely generated $k$-algebras (details can be found in [13], [8], [9]). If $V$ is a finite-dimensional vector subspace of $R$ and $n$ is a natural number, then $V^{n}$ denotes the vector subspace of $R$ generated by all $n$-fold products $v_{1} \cdots v_{n}$, where $v_{i} \in V$. It is understood that $V^{0}=k$. Define

$$
V^{(n)}=\sum_{i=0}^{n} V^{i}
$$

Now, assume that $R$ is finitely generated as $k$-algebra by $V$, i.e., $R=k[V]$. The Gelfand-Kirillov dimension of $R(\mathrm{GKdim}(R)$, for short) measures the asymptotic rate of growth of the "dimension function" $f(n)=\operatorname{dim}_{k} V^{(n)}$. In fact, $\operatorname{GKdim}(R)$ is the infimum of the real numbers $r$ such that $f(n) \leq n^{r}$ for $n \gg 0$. It is known that this value is independent of the choice of the generating subspace $V$. The GK dimension of a finitely generated left $R$-module $M$ is given by the growth of the function $f(n)=\operatorname{dim}_{k}\left(V^{(n)} U\right)$, where $U$ is any finite-dimensional vector subspace of $M$ that generates $M$ as left $R$-module ( $U$ is a generating vector subspace of $M$, for short). This dimension can be expressed by the following formula.

$$
\mathrm{GKdim}(M)=\lim \sup \log _{n} \operatorname{dim}_{k}\left(V^{(n)} U\right)
$$

Recall that the $k$-algebra $R$ is endowed with a multi-filtration $\left\{F_{\alpha}(R)\right\}$. Let $M$ be a multi-filtered left $R$-module with multi-filtration $\left\{F_{x}(M)\right\}$. We will assume that $F_{\alpha}(M), F_{z}(R)$ are vector subspaces of $M$ and $R$, respectively, for every multi-index $\alpha \in \mathbb{N}^{p}$.

Definition 2.1. A multi-index sequence for $M$ is a map $\alpha: \mathbb{N} \rightarrow \mathbb{N}^{p}$ such that

1. $\alpha(n+m) \leq \alpha(n)+\alpha(m)$, for every $n, m \in \mathbb{N}$.

2. $V^{(n)} U \subseteq F_{\alpha(n)}(M)$ for every $n \in \mathbb{N}$.

We can immediately deduce that $\alpha(n) \leq n \alpha(1)$ for every positive integer $n$. 
Example 2.2. The map $\alpha: \mathbb{N} \rightarrow \mathbb{N}^{p}$ given by

$$
\alpha(n)=\min \left\{\gamma \in \mathbb{N}^{p}: V^{(n)} U \subseteq F_{\gamma}(M) \text { and } V^{(n)} \subseteq F_{\gamma}(R)\right\}
$$

is a multi-index sequence for $M$.

Definition 2.3. The multi-filtration $\left\{F_{\alpha}(M)\right\}$ is said to be finite if $F_{\alpha}(M)$ is a vector space of finite dimension over $k$ for every $\alpha \in \mathbb{N}^{p}$.

If the multi-filtration $\left\{F_{a}(M)\right\}$ is finite, then it makes sense to define the sounding map for a given multi-index sequence $\{\alpha(n)\}$ by

$$
s(n)=\min \left\{j \in \mathbb{N}: F_{x(n)}(M) \subseteq V^{(n)} U\right\}
$$

Proposition 2.4. Let $\{\alpha(n)\}$ be any multi-index sequence for $M$. If the multi-filtrations on $R$ and $M$ are finite, then

$$
\operatorname{GKdim}(M) \leq \lim \sup \log _{n} \operatorname{dim}_{k}\left(F_{\alpha(n)}(M)\right)
$$

If, in addition, $F_{\rho}(R) F_{\sigma}(M)=F_{\rho+\sigma}(M)$ for every $\sigma, \rho \in \mathbb{N}^{p}$, then

$$
G \operatorname{Gim}(M)=\lim \sup \log _{n} \operatorname{dim}_{k}\left(F_{x(n)}(M)\right)
$$

Proof. The inequality is clear from the inclusions $V^{(n)} U \subseteq F_{a(n)}(M), n \in \mathbb{N}$. To obtain the equality, let $d$ be such that $F_{a(1)}(R) \subseteq V^{(d)}$. We will prove by induction on $n$ that $F_{z(n)}(M) \subseteq V^{(n-1) d+s(1))} U$. For $n=1$, the inclusion holds by (1). If $n>1$, then

$$
F_{x(n)}(M) \subseteq F_{n \times(1)}(M)=F_{\alpha(1)}(R) F_{(n-1) x(1)}(M) \subseteq V^{(d)} V^{((n-2) d+s(1))} U=V^{((n-1) d+s(1))} U
$$

This implies that $s(n) \leq d(n-1)+s(1)$. Hence, the asymptotic rate of growth of $\operatorname{dim}_{k}\left(F_{a(n)}(M)\right)$ is less or equal than that of $\operatorname{dim}_{k} V^{(n-1) d+s(1))} U$, which finishes the proof.

The foregoing result can be significantly improved in the graded case. Assume that $A=\oplus_{x \in \mathbb{N}^{p}} A_{\alpha}$ is an $\mathbb{N}^{p}$-graded $k$-algebra. This algebra is immediately multi-filtered by

$$
F_{x}(A)=\bigoplus_{\beta \leq \alpha} A_{\beta}
$$

Analogously, every $\mathbb{N}^{p}$-graded left $A$-module $M=\oplus_{\alpha \in \mathbb{N}^{p}} M_{\alpha}$ can be canonically viewed as a multi-filtered left module. For a nonzero homogeneous element $m \in M_{x}$, we will write $\operatorname{deg}(a)=\alpha$. Observe that if $a \in A$ and $m \in M$ are homogeneous elements such that $a m \neq 0$, then $\operatorname{deg}(a m)=\operatorname{deg}(a)+\operatorname{deg}(m)$. A vector subspace $X$ of $M$ is said to be homogeneous if $X$ has a basis consisting of homogeneous elements. 
Proposition 2.5. Let $M$ be a finitely generated graded left module over an $\mathbb{N}^{p}$-graded finitely generated k-algebra $A$. Assume that the filtrations induced on $M$ and $A$ by the gradings are finite. Let $\alpha_{0} \in \mathbb{N}^{p}$ be such that $A$ is generated as $k$-algebra by $V=F_{x_{0}}(A)$. Let $U$ be any finite-dimensional homogeneous generating subspace of $M$. If $\{\alpha(n)\}$ is any multi-index sequence for $M$, then

$$
\operatorname{GKdim}(M)=\lim \sup \log _{n} \operatorname{dim}_{k}\left(F_{x(n)}(M)\right)
$$

Proof. Let $s: \mathbb{N} \rightarrow \mathbb{N}$ be the sounding map for $\{\alpha(n)\}$. Every nonzero homogeneous element of $F_{\alpha(n)}(M)$ can be written as a sum of monomials of the form $v_{1} \cdots v_{t} u$, for homogeneous elements $v_{i} \in V, u \in U$ satisfying $v_{i} v_{i+1} \notin V$ and $t \leq s(n)$. There exists a nonzero homogeneous element $x \in F_{\alpha(n)}(M)$ such that $t=s(n)$ for at least one such monomial. Moreover, $\alpha(n) \geq \operatorname{deg}(x)=\operatorname{deg}\left(v_{1} \cdots v_{s(n)} u\right)$. Let $r=\left[\frac{5(n)}{2}\right]$, the integer part of $\frac{s(n)}{2}$. Then

$$
\begin{aligned}
n a(1) \geq \alpha(n) & \geq \operatorname{deg}\left(v_{1} \cdots v_{s(n)} u\right)=\operatorname{deg}\left(v_{1} \cdots v_{s(n)}\right)+\operatorname{deg}(u) \\
& \geq \operatorname{deg}\left(v_{1} v_{2}\right)+\cdots+\operatorname{deg}\left(v_{2 r-1} v_{2 r}\right)>\left[\frac{s_{n}}{2}\right] \alpha_{0} .
\end{aligned}
$$

If $d \alpha_{0}<\alpha(1)$ for every positive integer $d$, then

$$
V^{(d)} U \subseteq F_{d \alpha_{0}}(A) U \subseteq F_{x(1)} U
$$

for every $d$. This implies that $M$ is a finite-dimensional vector space and, thus, the equality (2) is clear. So we can assume that there is a positive integer $d$ such that $\alpha(1) \leq d \alpha_{0}$. By (3),

$$
\left[\frac{s(n)}{2}\right] a_{0}<n d \alpha_{0}
$$

whence

$$
\left[\frac{s(n)}{2}\right]<n d .
$$

This implies that $s(n) \leq 2 n d$ for every $n \in \mathbb{N}$ and, thus,

$$
\operatorname{dim}_{k}\left(V^{(n)} U\right) \leq \operatorname{dim}_{k} F_{x(n)}(M) \leq \operatorname{dim}_{k}\left(V^{(2 n d)} U\right)
$$

This implies that the polynomial growth of $\operatorname{dim}_{k}\left(V^{(n)} U\right)$ is the same as the polynomial growth of $\operatorname{dim}_{k}\left(F_{x(n)} M\right)$, as required.

Let us now return to the case of a multi-filtered left module $M$ over the multi-filtered $k$-algebra $R$. For any vector subspace $X$ of $M$, define 


$$
G(X)=\bigoplus_{\gamma \in \mathbb{N}^{p}} \frac{\left(X \cap F_{\gamma}(M)\right)+V_{y}(M)}{V_{\gamma}(M)}
$$

which is a vector subspace of $G(M)$.

Proposition 2.6. (i) Let $W$ be a vector subspace of $G(R)$ such that $G(R)=k[W]$. If $V$ is a vector subspace of $R$ such that $W \subseteq G(V)$, then $R=k[V]$.

(ii) Let $U$ be a generating subspace of $G(M)$. If $U^{\prime}$ is a subspace of $M$ such that $U \subseteq G\left(U^{\prime}\right)$, then $M=R U^{\prime}$.

Proof. Let $r \in R$. If $r \neq 0$, then let $\alpha$ be the first element of $\mathbb{N}^{p}$ such that $r \in F_{\alpha}(R)$. Then $r+V_{a}(R) \in G(R)=k[W]$ and, therefore, there is $i \geq 0$ such that $r+V_{\alpha}(R) \in W^{(i)}$. Now, it is not difficult to show that $G(V)^{(i)} \subseteq G\left(V^{(i)}\right)$. Hence, there is $t \in V^{(i)}$ such that $r-t \in V_{x}(R)$. If $r-t \neq 0$, then let $\beta$ be minimum with $r-t \in F_{\beta}(R)$. It is clear that $\beta<\alpha$. This entails that after finitely many steps we must obtain that $r \in V^{(j)}$ for some positive integer $j$. The proof of the second part is similar.

Lemma 2.7. If $X$ is a finite-dimensional vector subspace of $M$, then $\operatorname{dim}_{k} G(X)=\operatorname{dim}_{k} X$.

Proof. Assume that $X \neq\{0\}$. Define recursively a finite increasing sequence

$$
\beta_{-1}<\beta_{0}<\beta_{1}<\cdots<\beta_{m}
$$

as follows. We will consider $F_{-\infty}(M)=\{0\}$ and $\beta_{-1}=-\infty$. For $i \geq 0$, define

$$
\beta_{i}=\inf \left\{\beta \in \mathbb{N}^{p} \mid X \cap F_{\beta_{i-1}}(M) \subsetneq X \cap F_{\beta}(M)\right\}
$$

This gives a strictly increasing sequence of vector subspaces of $X$

$$
\{0\} \subsetneq X \cap F_{\beta_{0}}(M) \subsetneq \cdots \subsetneq X \cap F_{\beta_{m}}(M)=X .
$$

Easy computations show that

$$
G(X) \cong \bigoplus_{i=0}^{m} \frac{X \cap F_{\beta_{i}}(M)}{X \cap F_{\beta_{i-1}}(M)}
$$

Now the lemma follows easily.

Theorem 2.8. Let $M$ be a multi-filtered left module over the multi-filtered $k$-algebra $R$. Assume $G(R)$ is a finitely generated $k$-algebra and that $G(M)$ is finitely generated as a left $G(R)$-module. Then

$$
\mathrm{GK} \operatorname{dim}\left({ }_{R} M\right) \geq \mathrm{GK} \operatorname{dim}\left({ }_{G(R)} G(M)\right)
$$


If, in addition, the multi-filtrations are finite, then

$$
\mathrm{GK} \operatorname{dim}\left({ }_{R} M\right)=\mathrm{GK} \operatorname{dim}\left({ }_{G(R)} G(M)\right) .
$$

Proof. We will first prove the inequality. Let $W$ be a homogeneous finitedimensional subspace of $G(R)$ such that $G(R)=k[W]$. It is easy to see that there is a finite-dimensional subspace $V$ of $R$ such that $W \subseteq G(V)$. By Proposition 2.6, $R=k[V]$. Analogously, given a finite-dimensional generating subspace $U$ of $G(M)$, choose an f.d. generating vector subspace $U^{\prime}$ of $M$ such that $U \subseteq G\left(U^{\prime}\right)$. For every natural number $n$,

$$
W^{(n)} U \subseteq G(V)^{(n)} G\left(U^{\prime}\right) \subseteq G\left(V^{(n)}\right) G\left(U^{\prime}\right) \subseteq G\left(V^{(n)} U^{\prime}\right) .
$$

Thus, $\operatorname{dim}_{k} W^{(n)} U \leq \operatorname{dim}_{k} G\left(V^{(n)} U^{\prime}\right)$. By Lemma 2.7,

$$
\operatorname{dim}_{k} G\left(V^{(n)} U^{\prime}\right)=\operatorname{dim}_{k} V^{(n)} U^{\prime},
$$

which entails that $\mathrm{GK} \operatorname{dim}\left({ }_{R} M\right) \geq \mathrm{GK} \operatorname{dim}(G(R) G(M))$.

Now, we will prove the equality in the "finite" case. The filtration $\left\{F_{\alpha}(R)\right\}$ is finite, which implies, by Lemma 2.7, that the canonical filtration on $G(R)$ is also finite. Since $G(R)$ is a finitely generated $k$-algebra, there is $\alpha_{0} \in \mathbb{N}^{p}$ such that $W=F_{x_{0}}(G(R))$ generates $G(R)$ as algebra. Since $G(M)$ is finitely generated, there is $U=F_{y_{0}}(G(M)$ ) such that $U$ generates $G(M)$ as $G(R)$-module. Put $V=F_{x_{0}}(R)$ and $U^{\prime}=F_{\gamma_{0}}(M)$. It is clear that $G(V)=W$ and $G\left(U^{\prime}\right)=U$. By Proposition 2.6, $R$ is generated as a $k$-algebra by $V$ and $M$ is generated as an $R$-module by $U^{\prime}$. Let $\{\alpha(n)\}$ be a multi-index sequence for $M$ (choose, e.g., the sequence given in Example 2.2). First, we will prove that it is also a multi-index sequence for $G(M)$. By (4), $W^{(n)} U \subseteq G\left(V^{(n)} U^{\prime}\right)$. The inclusion

$$
G\left(V^{(n)} U^{\prime}\right) \subseteq G\left(F_{\alpha(n)}(M)\right)=F_{\alpha(n)}(G(M))
$$

tells us that $\{\alpha(n)\}$ is a multi-index sequence for $G(M)$. By Proposition 2.5,

$$
\text { GK } \operatorname{dim}(G(R) G(M))=\text { lim sup } \log _{n} \operatorname{dim}_{k} G\left(F_{\alpha(n)}(M)\right) \text {. }
$$

By Lemma 2.7, $\operatorname{dim}_{k} G\left(F_{x(n)}(M)\right)=\operatorname{dim}_{k} F_{x(n)}(M)$ for every $n \in \mathbb{N}$. This, together with (5), gives

$$
\text { GK } \operatorname{dim}\left(G_{G(R)} G(M)\right)=\text { limsup } \log _{n} \operatorname{dim}_{k}\left(F_{\alpha(n)}(M)\right) .
$$

By Proposition 2.4, lim sup $\log _{n} \operatorname{dim}_{k}\left(F_{x(n)}\right) \geq G K \operatorname{dim}(M)$. This finishes the proof.

Remark 2.9. The proof of Theorem 2.8 gives a procedure to obtain a multi-index sequence $\{\alpha(n)\}$ for $M$ such that

$$
\mathrm{GK} \operatorname{dim}(M)=\lim \sup \log _{n} \operatorname{dim}_{k}\left(F_{\alpha(n)}(M)\right) .
$$




\section{GELFAND-KIRILLOV DIMENSION OF MULTI-FILTERED ALGEBRAS 163}

Choose $\alpha_{0}, \gamma_{0} \in \mathbb{N}^{p}$ such that $F_{x_{0}}(G(R))$ generates $G(R)$ as a $k$-algebra and $F_{\gamma_{0}}(G(M))$ generates $G(M)$ as a $G(R)$-module. Then any multi-index sequence $\{\alpha(n)\}$ for $\left\{F_{\alpha}(M)\right\}$ with $V=F_{x_{0}}(R)$ and $U^{\prime}=F_{\gamma_{0}}(M)$ works.

Theorem 2.10. Assume that the multi-filtration on $R$ is finite and that $F_{0}(R)=k$. If $G(R)$ is a left noetherian finitely-generated k-algebra, then the Gelfand-Kirillov dimension is exact on short exact sequences of finitely-generated left $R$-modules.

Proof. Let $M$ be a finitely generated left $R$-module, and $L$ be a submodule of $M$. Set $N=M / L$. We have to prove that

$$
G K \operatorname{dim}(M)=\max \{G K \operatorname{dim}(L), G K \operatorname{dim}(N)\} .
$$

By Theorem 1.5, $R$ is left noetherian and, therefore, $L$ is finitely generated. Let $X$ be a finite-dimensional generating vector subspace of $L$ and choose a generating subspace of finite dimension $U$ of $M$ such that $X=L \cap U$. Endow $M$ with the finite multifiltration given by $F_{x}(M)=F_{x}(R) U$, for every $\alpha \in \mathbb{N}^{p}$. Since $F_{0}(R)=k$, we have that $F_{0}(M)=U$ and, therefore, $G(M)_{0}=U$. It is easy to prove that $G(M)$ is generated by $U$. Let $\alpha_{0} \in \mathbb{N}^{p}$ be such that $F_{\alpha_{0}}(G(R))$ generates $G(R)$ as $k$-algebra, and put $\gamma_{0}=0$. Choose a multi-index sequence $\{\alpha(n)\}$ according to Remark 2.9. For every $\alpha \in \mathbb{N}^{p}$, set $F_{\alpha}(L)=L \cap F_{x}(M)$ and $F_{x}(N)=\left(L+F_{\alpha}(M)\right) / L$. In this way, we obtain multi-filtrations on $L$ and $N$ which are, obviously, finite. Moreover, the exact sequence of left $R$ modules

$$
0 \rightarrow L \longrightarrow M \rightarrow N \rightarrow 0
$$

induces an exact sequence of graded left $G(R)$-modules

$$
0 \longrightarrow G(L) \longrightarrow G(M) \longrightarrow G(N) \longrightarrow 0 .
$$

Since ${ }_{G(R)} G(M)$ is finitely generated and $G(R)$ is left noetherian, $G(L)$ and $G(N)$ are finitely generated. Finally, $\{\alpha(n)\}$ is a multi-index sequence for both $L$ and $N$ in the conditions of Remark 2.9 (with $\gamma_{0}=\mathbf{0}$ ). The equality (6) is now deduced from Remark 2.9 and the exactness of the sequence of vector spaces

$$
0 \longrightarrow F_{x(n)}(L) \longrightarrow F_{z(n)}(M) \longrightarrow F_{\alpha(n)}(N) \longrightarrow 0
$$

for every natural number $n$.

Definition 2.11 (McConnell, [12]). A $k$-algebra $A$ is called semi-commutative if $A$ has a set of $k$-algebra generators $x_{1}, \ldots, x_{n}$ such that, for each $i, j, x_{i} x_{j}=q_{i j} x_{j} x_{i}$, where $0 \neq q_{i j} \in k$.

Corollary 2.12. Let $M$ be a multi-filtered left $R$-module over a multi-filtered $k$-algebra 
$R$ such that $G(M)$ is finitely generated over $G(R)$. Assume that the filtrations are finite and that $G(R)$ is semi-commutative. Then $G \mathrm{GKdim}\left({ }_{R} M\right)=\mathrm{GK} \operatorname{dim}\left({ }_{G(R)} G(M)\right.$ ) is an integer. Moreover, if $F_{0}(R)=k$, then the Gelfand-Kirillov dimension is exact on short exact sequences of finitely generated left $R$-modules.

\section{Examples}

This final section is devoted to providing some non-trivial examples of multi-filtered algebras by finite filtrations having a semi-commutative associated graded ring.

Definition 3.1. An admissible order $\leq$ on $\mathbb{N}^{p}$ is called locally finite if the interval $[0, \alpha]=\left\{\gamma \in \mathbb{N}^{p}: \gamma \leq \alpha\right\}$ is finite for every $\alpha \in \mathbb{N}^{p}$.

Proposition 3.2. Let $R$ be a k-algebra finitely generated by $x_{1}, \ldots, x_{p}$ and set $X^{\alpha}=x_{1}^{\alpha_{1}} \ldots x_{p}^{\alpha_{p}}$ for all $\alpha \in \mathbb{N}^{p}$. If there is a locally finite admissible order ' $\leq$ ' and nonzero scalars $q_{j i} \in K$ such that

$$
x_{j} x_{i}=q_{j i} x_{i} x_{j}+\sum_{\gamma<\epsilon_{i}+\epsilon_{j}} c_{y} X^{\gamma}
$$

where $\epsilon_{i}=(0, \ldots, 1, \ldots, 0)$, then

(i) $R$ is endowed with a finite filtration given by

$$
F_{\alpha}(R)=\sum_{\beta \leq a} k X^{\beta}
$$

(ii) $G(R)$ is a semi-commutative algebra with finite associated filtration. In particular, Corollary 2.12 applies.

Proof. Let $\alpha, \beta \in \mathbb{N}^{p}$. By [3, Proposition 1.7] (see also [7, Lemma 1.4]), there exists a nonzero scalar $q_{\alpha, \beta}$ in $k$ such that

$$
X^{\alpha} X^{\beta}=q_{\alpha, \beta} X^{\alpha+\beta}+\sum_{\gamma<\alpha+\beta} c_{\gamma} X^{\gamma}
$$

From this we get that $\operatorname{dim}_{k} F_{a}(R)$ is less than or equal to the cardinal of the finite interval $[0, \alpha]$ and that $\left\{F_{\alpha}\right\}$ is indeed a multi-filtration. It is clear that $G(R)$ is semicommutative.

Finally, we will exhibit some "non classical" examples of algebras satisfying the hypotheses of Proposition 3.2. We will use "weighted" admissible orders on $\mathbb{N}^{p}$, defined as follows. Let $\mathbf{w}=\left(w_{1}, \ldots, w_{p}\right)$ be a vector in $\mathbb{R}^{p}$ with $w_{i}>0$ for every $i=1, \ldots, p$. Consider the usual dot product $(-,-)$ on $\mathbb{R}^{p}$. By $<_{\text {lex }}$ we will denote the lexicographic 
order in $\mathbb{N}^{p}$ with $\epsilon_{1}<\epsilon_{2}<\cdots<\epsilon_{p}$. The binary relation in $\mathbb{N}^{p}$ defined for $\alpha, \beta \in \mathbb{N}^{p}$ as $\alpha<_{w} \beta$ if $\langle\alpha, w\rangle<\langle\beta, w\rangle$ or $\langle\alpha, w\rangle=\langle\beta, w\rangle$ and $\alpha<_{\text {lex }} \beta$ is a locally finite admissible order in $\mathbb{N}^{p}$. We will say that $w$ is the weight vector for the order $<_{m}$.

Example 3.3. Let $R=k\left[x_{1}\right]\left[x_{2} ; \sigma_{2}, \delta_{2}\right] \ldots\left[x_{p} ; \sigma_{p}, \delta_{p}\right]$ be an iterated Ore extension of $k$. Assume that $\sigma_{j}\left(x_{i}\right)=q_{i j} x_{i}$, for every $i<j \leq p$, where the $q_{i j}$ 's are nonzero scalars in $k$. The set $\mathcal{B}=\left\{X^{\alpha} ; \alpha \in \mathbb{N}^{p}\right\}$ is a $k$-basis of $R$. We retain here the notation of Proposition 3.2. For every $p=\sum_{x \in \mathbb{N}^{p}} c_{z} X^{z}$, set $N(p)=\left\{\alpha \in \mathbb{N}^{p} \mid c_{z} \neq 0\right\}$. We will construct a weight vector $\mathbf{w}=\left(w_{1}, \ldots, w_{p}\right)$. Set $w_{1}=1$ and define $w_{12}$ as the degree in $x_{1}$ of the polynomial $\delta_{2}\left(x_{1}\right)$. Put $w_{2}=\max \left\{1, w_{12}\right\}$. Suppose we have defined $w_{1}, \ldots, w_{j-1}$ for $j \geq 2$. For $k=1, \ldots, j-1$, set $w_{k j}=\max \left\{\alpha_{1} w_{1}+\cdots+\alpha_{j-1} w_{j-1} ; \alpha \in N\left(\delta_{j}\left(x_{k}\right)\right)\right\}$ and choose $w_{j}=\max \left\{1, w_{k j}-w_{i} ; 1 \leq i, k \leq j-1\right\}$. Endow $\mathbb{N}^{p}$ with the admissible order $<_{w}$. If $i<j \leq q$, then $\left(\epsilon_{i}+\epsilon_{j}, w\right\rangle=w_{i}+w_{j} \geq w_{i j} \geq\langle\alpha, w\rangle$ for every $\alpha \in N\left(\delta_{j}\left(x_{i}\right)\right)$. Therefore, $\alpha<_{n} \epsilon_{i}+\epsilon_{j}$ for every $\alpha \in N\left(\delta_{j}\left(x_{i}\right)\right)$ whenever $i<j$. By Proposition 3.2, $R$ has a finite multi-filtration with semi-commutative associated graded ring. In particular, the Gelfand-Kirillov dimension is exact on sequences of finitely generated modules. Some examples of these iterated Ore extensions are the algebras $H(p, \lambda)$ defined in [1], which include the quantum co-ordinate algebras of $M_{n}(k)$, and the multiparameter quantized Weyl algebra $R=A_{n}^{Q, \mathrm{r}}(k)$ introduced in [11] (see also [5]). The iterated differential operator algebras of [17] are also covered.

Example 3.4. Our last example is the quantized universal enveloping algebra $U_{q}(\mathfrak{s l}(v+1))$, with $q \in k$ non-zero and $q^{8} \neq 1$. We will follow, essentially, the presentation of this algebra given in [18] and [12]. For our purposes, it is better to consider $R=U_{q}(\mathfrak{s l}(v+1))$ as a homomorphic image of the algebra $S=V_{q}(\operatorname{sl}(v+1))$ defined as follows. Order $\mathbb{N}^{2}$ lexicographically, i.e., $(i, j)<(m, n)$ if $i<m$ or $i=m$ and $j<n$. There are six possible ways to have $(i, j)<(m, n)$ :
I. $i=m<j<n$,
II. $i<m<n<j$,
III. $i<m<j=n$,
IV. $i<m<j<n$,
V. $i<j=m<n$,
VI. $i<j<m<n$.

The algebra $S$ is generated by $f_{i j}, K_{i}, L_{i}, e_{i j}$, for $1 \leq i<j \leq v+1$, where $K_{i}, L_{i}$ are semi-commutating variables and the relations for the variables $f_{i j}, e_{i j}$ are described as follows. For $(i, j)<(m, n)$,

I or III. $e_{i j} e_{m n}=q^{2} e_{m n} e_{i j}$,

II or VI. $e_{i j} e_{m n}=e_{m n} e_{i j}$,

IV. $\left[e_{i j}, e_{m n}\right]=\left(q^{2}-q^{-2}\right) e_{i n} e_{m j}$, 
V. $q e_{i m} e_{m n}-q^{-1} e_{m n} e_{i m}=e_{i n}$,

and similar relations among the $f_{i j}$ (here, $[x, y]=x y-y x$ ). The relations between the $e_{i j}$ and $f_{i j}$ are:

$$
\begin{aligned}
& {\left[e_{i j}, f_{m n}\right]= \begin{cases}(-1)^{j-i+1} q f_{j n} K_{i}^{2} \ldots K_{j-1}^{2} & (\text { case I) } \\
(-1)^{n-m+1} q K_{m}^{2} \ldots K_{j-1}^{2} e_{i m} & \text { (case III) } \\
(-1)^{j-m+1}\left(q^{4}-1\right) f_{j n} K_{m}^{2} \ldots K_{j-1}^{2} e_{i m} & \text { (case IV) } \\
0 & \text { (cases II, V, VI) }\end{cases} } \\
& {\left[e_{m n}, f_{i j}\right]= \begin{cases}(-1)^{j-i} q^{-1} L_{i}^{2} \ldots L_{j-1}^{2} e_{i n} & \text { (case I) } \\
(-1)^{n-m} q^{-1} f_{i m} L_{m}^{2} \ldots L_{n-1}^{2} & \text { (case III) } \\
(-1)^{j-m}\left(1-q^{-4}\right) f_{i m} L_{m}^{2} \ldots L_{j-1}^{2} e_{j n} & \text { (case IV) } \\
0 & \text { (cases II, V, VI) }\end{cases} }
\end{aligned}
$$

Finally,

$$
\left[e_{i j}, f_{i j}\right]=(-1)^{j-i+1} \frac{K_{i}^{2} \ldots K_{j-1}^{2}-L_{i}^{2} \ldots L_{j-1}^{2}}{\left(q^{2}-q^{-2}\right)} .
$$

It follows from [18, Section 3] that $R$ is a homomorphic image of $S$ under the mapping $f_{i j} \mapsto f_{i j}, e_{i j} \mapsto e_{i j}, K_{i} \mapsto K_{i}$ and $L_{i} \mapsto K_{i}^{-1}$. We will endow $S$ with a finite multifiltration. Order the variables

$$
f_{12}<\cdots<f_{v v+1}<K_{1}<\cdots<K_{v}<L_{1}<\cdots<L_{v}<e_{12} \cdots<e_{v v+1}
$$

where the $f_{i j}$ and the $e_{i j}$ are ordered by the lexicographic order in $\mathbb{N}^{2}$. For $(i, j) \in \mathbb{N}^{2}$, put $w_{i j}=i+3 j$, and consider the weight vector in $\mathbb{R}^{v^{2}+3 v}$

$$
\mathbf{w}=(w_{12}, \ldots, w_{v v+1}, \overbrace{1, \ldots, 1}^{2 v} w_{12}, \ldots, w_{v v+1})
$$

where the subindices of the first $(v+1) v / 2$ and the last $(v+1) v / 2$ components are ordered lexicographically. Assign to any "standard" word in $S$

$$
f_{12}^{a_{12}} \ldots f_{v v+1}^{a_{w+1}} K_{1}^{k_{1}} \ldots K_{v}^{k_{v}} L_{1}^{h_{1}} \ldots L_{v}^{l_{v}} e_{12}^{b_{12}} \ldots e_{v v+1}^{b_{w+1}}
$$

the multi-index

$$
\alpha=\left(a_{12}, \ldots, a_{v v+1}, k_{1}, \ldots, k_{v}, l_{1}, \ldots, l_{v}, b_{12}, \ldots, b_{w+1}\right) .
$$

Some straightforward computations show that $S$ is as in the hypotheses of Proposition 3.2 with respect to the order $<_{\pi}$ in $\mathbb{N}^{v^{2}+3 v}$. Therefore, this algebra can be endowed with a finite multi-filtration $\left\{F_{a}(S)\right\}$ in such a way that the associated graded algebra is 


\section{GELFAND-KIRILLOV DIMENSION OF MULTI-FILTERED ALGEBRAS 167}

isomorphic to a semi-commutative algebra. Now, let $\varphi: S \rightarrow R$ be the afore-mentioned surjective homomorphism of algebras. This map $\varphi$ induces a finite multi-filtration on the quantized universal enveloping algebra by defining, for every $\alpha \in \mathbb{N}^{2^{2}+3 v}$, $F_{a}(R)=\varphi\left(F_{z}(S)\right)$. Moreover, the associated multi-graded ring $G(R)$ is a homomorphic image of $G(S)$ and, thus, it is semi-commutative.

\section{REFERENCES}

1. M. Artin, W. Schelter and J. TAte, Quantum deformations of $G L_{n}$, Comm. Pure Appl. Math. 44 (1991), 879-895.

2. T. BECKER and V. WEISPFENNING, Gröbner basis. A computational approach to commutative algebra (Springer, New York, 1993).

3. J. L. Bueso, F. J. Castro, J. Gómez Torrecillas and F. J. Lobillo, An introduction to effective calculus in quantum groups, in Rings, Hopf algebras and Brauer groups (S. Caenepeel and A. Verschoren, eds., Marcel Dekker, 1998), 55-83.

4. C. De Concini and C. Procesi, Quantum groups, in D-Modules, Representation Theory and Quantum Groups (G. Zampieri and A. D'Agnolo, eds., Lecture Notes in Math., 1565, Springer, 1993), 31-140.

5. K. R. Goodearl and T. H. Lenagan, Catenarity in quantum algebras, J. Pure Appl. Algebra 111 (1996), 123-142.

6. A. JOSEPH, Quantum groups and their primitive ideals (Springer, New York, 1995).

7. A. Kandri-Rody and V. WeISPFEnNing, Non-Commutative Gröbner basis in algebras of solvable type, J. Symbolic Comput. 9 (1990), 1-26.

8. G. R. Krause and T. H. Lenagan, Growth of algebras and Gelfand-Kirillov dimension (Research Notes in Mathematics, 116, Pitman Pub. Inc., London, 1985).

9. M. LORENZ, Gelfand-Kirillov dimension and Poincaré series (Cuadernos de Algebra, 7, Universidad de Granada, 1988).

10. G. Luszrig, Canonical bases arising from quantized enveloping algebras, J. Amer. Math. Soc. 3 (1990), 447-498.

11. G. Maltsiniotis, Calcul difféntiel quantique (Université de Paris, VII, 1992).

12. J. C. MCConnell, Quantum groups, filtered rings and Gelfand-Kirillov dimension, in Noncommutative Ring Theory (Lecture Notes in Math., 1448, Springer, 1990), 139-149.

13. J. C. McConnell and J. C. Robson, Noncommutative Noetherian Rings (J. Wiley and Sons, Chichester-New York, 1988).

14. J. C. McConnell and J. T. Stafford, Gelfand-Kirillov dimension and associated graded modules, J. Algebra 125 (1989), 179-214.

15. T. MORA, Seven variations on standard bases (Univ. Genova, 1988), preprint.

16. L. Robbiano, On the theory of graded structures, J. Symbolic Comput. 2 (1986), 139-170. 
17. G. Sigurdsson, Ideals in universal enveloping algebras of solvable lie algebras, Comm. Algebra 15 (1987), 813-826.

18. H. Yamane, A Poincare-Birkhoff-Witt Theorem for quantized enveloping algebra of type $A_{N}$, Publ. RIMS. Kyoto Univ. 25 (1989), 503-520.

Dept. Algebra

Facultad de Ciencias

UNIVERSIDAD DE GRANADA

E-18071 GRANADA

SPAIN

E-mail address: torrecil@ugr.es 